\title{
Analytical model for gyro-phase drift arising from abrupt inhomogeneity
}

\author{
Jeffrey J. Walker ${ }^{1} \dagger$, M. E. Koepke ${ }^{1}$, M. I. Zimmerman ${ }^{2}$, W. M. Farrell ${ }^{2}$ \\ and V. I. Demidov ${ }^{1,3}$ \\ ${ }^{1}$ Department of Physics, West Virginia Univeristy, Morgantown WV 26506, USA \\ ${ }^{2}$ Goddard Space Flight Center, National Aeronautics and Space Administration, \\ Greenbelt MD 20771, USA \\ ${ }^{3}$ University ITMO, Kronverkskiy pr. 49, St. Petersburg 197101, Russia
}

(Received 9 September 2013; revised 7 October 2013; accepted 16 October 2013; first published online 13 December 2013)

If a magnetized-orbit-charged grain encounters any abrupt inhomogeneity in plasma conditions during a gyro-orbit, such that the resulting in-situ equilibrium charge is significantly different between these regions $\left(q_{1} / q_{2} \sim 2\right.$, where $q_{1}$ is the in-situ equilibrium charge on one side of the inhomogeneity, $q_{2}$ is the in-situ equilibrium charge on the other side, and $q_{1}<q_{2}<0$ ), then the capacitive effects of charging and discharging of the dust grain can result in a modification to the orbit-averaged grain trajectory, i.e. gyro-phase drift. The special case of $q_{1} / q_{2}$ is notioned for the purpose of illustrating the utility of the method. An analytical expression is derived for the grain velocity, assuming a capacitor approximation to the OML charging model. For cases in which a strong electric field suddenly appears in the wake or at the space-plasma-to-crater interface from solar wind and/or ultraviolet illumination and in which a magnetic field permeates an asteroid, comet, or moon, this model could contribute to the interpretation of the distribution of fields and particles.

\section{Introduction to Gyro-phase drift}

Gyro-phase drift is a guiding center drift that is directly dependent on the charging rate limit of dust grains (Northrop and Hill 1983). The effect of introducing a gyrophase dependence on the grain charge leads to two orthogonal components of guiding center drift. One component, referred to here as grad-q drift $\left(\boldsymbol{v}_{\nabla q}\right)$, results from the time-varying, gyro-angle-dependent in-situ equilibrium grain charge, assuming that the grain charging is instantaneous. For this component, the grain is assumed to be always in its in-situ equilibrium charge state and this state gyro-synchronously varies with respect to the grain's average charge state. The other component, referred to here as the gyro-phase drift, arises from any non-instantaneous-charging-induced modification of the guiding center drift and points in the direction of $-\nabla R_{L d}$ (where $R_{L d}$ is the grain gyro-radius), i.e. the direction associated with increasing magnitude of in-situ equilibrium charge state. Koepke et al. (2013) simulated the effects of a gradual inhomogeneity on the magnitude and direction of gyro-phase drift velocity. Here we arrive at an analytical expression for the magnitude and direction of gyro-phase drift velocity for the case of abrupt inhomogeneity. 
When there is an abrupt inhomogeneity in the plasma conditions, the in-situ equilibrium grain charge state will be different on either side of the inhomogeneity. If a magnetized-orbit dust grain gyrates through the inhomogeneity, the grain changes charge state $q$ during its gyro-orbit by either gaining electrons, thus attaining a more negative charge state, or losing electrons, thus attaining a less negative charge state. The function $q(t)$ represents the time-dependent charge of the dust grain.

For the Orbit Motion Limited (OML) model, Goree (1994) describes a method for characterizing the charging time of dust grains from zero elementary charges to one e-folding of the in-situ equilibrium value in a homogeneous plasma as $\tau_{c h}=$ $k_{T} T_{e}^{1 / 2} /\left(a n_{0}\right)$, where $T_{e}$ is the electron temperature in $\mathrm{eV}, a$ is the dust grain radius in meters, $n_{0}$ is the plasma density in $\mathrm{m}^{-3}$, and $k_{T}$, in units of seconds $\mathrm{m}^{-2} \mathrm{eV}^{-\frac{1}{2}}$, is a non-trivial function of both $T_{i} / T_{e}$ and $m_{i} / m_{e}$ that is fit to a numerical model. It is apparent from this expression that the charging time increases with electron temperature, and is inversely proportional to the grain size and background plasma density.

The method of Goree (1994) can be adapted to the inhomogeneous case of two neighboring homogeneous regions, each with its own distinct equilibrium charge state of the grain. Represent the two equilibrium charge states of the grain on either side of the abrupt inhomogeneity by $q_{1}$ and $q_{2}$, with $q_{1}<q_{2}<0$. The discharging time is the time elapsed between $q(t=0)=q_{1}$ and $q\left(t=\tau_{d}\right)=q_{1}+\frac{1}{\mathrm{e}}\left(q_{1}-q_{2}\right)$, where 'e' represents the base of the natural logarithm. It is possible that $q(t)$ will not reach the in-situ equilibrium charge state $q_{2}$ by the end of the discharging part of the gyro-orbit at time $t=t_{d f}$. The charging time is the time elapsed between $q\left(t_{d f}\right)=q_{d f}$ and $q\left(t=t_{d f}+\tau_{c}\right)=q_{d f}+\frac{1}{\mathrm{e}}\left(q_{d f}-q_{1}\right)$. These charging time, obtained from OML, can fit to a discharging/charging capacitor model and the function $q(t)$ can be prescribed for the discharging/charging parts of the gyro-cycle, as shown later in (5.2) and (5.3). The procedure is represented graphically in Fig. 2. In principle, the effect of repeated excursions between abrupt inhomogeneous plasma regions is straightforward to analyze, although here we focus on the first excursion.

\section{Parameters, inhomogeneity, and charge model}

To demonstrate the dependence of gyro-phase drift on charging rate, an abrupt inhomogeneity in ultraviolet (UV) illumination is selected (UV-present for $x>0$, UV-absent for $x<0$ ) as the abrupt inhomogeneous condition of convenience, while all other plasma parameters are the same on either side of this inhomogeneity. In the presence of $\mathrm{UV}$, a photoelectron current exists that will cause the dust grain to charge less negatively than in the absence of UV. Here the photoelectric current $I_{\mathrm{ph}}$ is arbitrarily imposed so that $q_{1} / q_{2} \sim 2$. The ratio $q_{1} / q_{2} \sim 2$, while notional, may be possible using a UV source similar to Dove et al. (2012) in conjunction with the Magnetized Dusty Plasma Experiment (Thomas et al. 2012).

An abrupt inhomogeneity in plasma temperature, density, or other parameters could also be applied in a similar fashion to this investigation, but an abrupt inhomogeneity in UV illumination is a readily conceivable scenario in a space or laboratory setting, such as the experiments of Sickafoose et al. (2000). For simplicity in the analysis, uniform electric and magnetic fields $(\boldsymbol{E}=0$ and $\boldsymbol{B}=B \hat{z}$, with $B=4 \mathrm{~T}$, respectively) are chosen so that only grad-q and gyro-phase drifts arise and so no other diamagnetic drifts occur. A magnetic field of $4 \mathrm{~T}$ is achievable in the Magnetized Dusty Plasma Experiment (Thomas et al. 2012). Isotropic UV illumination is assumed to prevent electron recoil and radiation forces as suggested by Rosenberg and Mendis (1995). 
Only the motion in the $x-y$ plane is considered, and $a=0.05 \mu \mathrm{m}$ grains have no velocity along the magnetic field direction $\left(v_{z}(t)=0\right)$. The dust grain is started at the position $x(t=0)=0, y(t=0)=0$ so that it encounters the transition region $(x=0)$ of this inhomogeneity at least twice during the first gyro-orbit. The dust grain starts with velocity components $v_{x}(t)=v_{\perp}$ and $v_{y}(t)=0$, where $v_{\perp}=0.124 \mathrm{~ms}^{-1}$ is the grain velocity perpendicular to the magnetic field. We demonstrate for the slab model that $v_{\perp}$ is not a crucial parameter, so $v_{\perp}=\sqrt{\frac{2 k_{b} T_{N}}{m_{d}}}$, where $k_{b}$ is the Boltzmann constant, $T_{N}=0.025 \mathrm{eV}$ is the neutral gas temperature, and $m_{d}$ is the dust grain mass. All parameters of the problem are chosen so that $V_{d}$, the dust surface potential relative to the local space potential or $V_{\text {space }}-V_{\text {surface }}$ is always negative.

For our choice of parameters in this paper, the gyro-radii of electrons and ions are expected to satisfy $R_{L e}, R_{L i}>a$, so the unmagnetized OML, or OML currents (Allen 1992) are used. For Maxwellian populations of electrons and ions, and dust grain velocities much less than the ion thermal speed and $q<0$, these currents are:

$$
\begin{gathered}
I_{e}=-e 4 \pi a^{2} n_{e} \frac{v_{\text {the }}}{2 \sqrt{\pi}} \exp \left(\frac{e q}{C k_{b} T_{e}}\right), \\
I_{i}=e 4 \pi a^{2} n_{i} \frac{v_{\text {thi }}}{2 \sqrt{\pi}}\left(1-\frac{e q}{C k_{b} T_{i}}\right), \\
I_{\mathrm{ph}}=e \pi a^{2} f,
\end{gathered}
$$

where $e$ is the elementary charge, $f$ represents the flux of photoelectrons for a given UV source and grain material, $v_{\text {the }}=\sqrt{\frac{2 k_{b} T_{e}}{m_{e}}}, v_{\text {thi }}=\sqrt{\frac{2 k_{b} T_{i}}{m_{i}}}, n_{e}=n_{i}=n_{0}$ because the plasma is treated as dust-absent, $V_{d}=q / C$, and $C=4 \pi \epsilon_{0} a$ is the grain capacitance. $T_{e}$ and $T_{i}$ represent electron and ion temperature respectively, while $m_{e}$ and $m_{i}$ represent electron and ion mass respectively. For the portion of the grain's gyro-orbit that is illuminated by UV $(x>0)$, the magnitude of the photoelectric current of electrons is non-zero, while it is identically zero when it is shadowed, or $x<0$. The total current on either side of the inhomogeneity can be expressed as:

$$
\begin{gathered}
I_{\mathrm{tot}}=I_{e}+I_{i}+I_{\mathrm{ph}} \text { for } x>0, \\
I_{\mathrm{tot}}=I_{e}+I_{i} \text { for } x<0 .
\end{gathered}
$$

Equation (2.2) describes $I_{\text {tot }}$ when UV is present, and (2.3) describes $I_{\text {tot }}$ when UV is absent. The photoelectric current in the UV-present region causes the dust grain to reach a less negative in-situ equilibrium charge state $q_{2}$ compared with the in-situ equilibrium charge state $q_{1}$ in the UV-absent region.

The photocurrent $I_{\mathrm{ph}}$ is determined by

$$
I_{\mathrm{ph}}=e \pi a^{2} f=e \pi a^{2} \int_{0}^{\lambda_{1}} F(\lambda) \chi(\lambda) \mathrm{d} \lambda,
$$

where $F(\lambda)$ is the flux of UV photons as a function of wavelength $\lambda$ for a UV source, $\chi(\lambda)$ is the quantum efficiency of photoemission for the grain material, and $\lambda_{1}$ is the wavelength cutoff, representing the longest wavelength capable of producing a photoelectron for a given work function (Colwell et al. 2005). In order to satisfy $q_{1} / q_{2} \sim 2$ for the $a=0.05-\mu \mathrm{m}$ grain in a plasma with background density $n_{0}=$ $10^{13} \mathrm{~m}^{-3}$ and electron, ion temperatures $T_{e}=1.6 \mathrm{eV}, T_{i}=0.025 \mathrm{eV}, I_{\mathrm{ph}} \sim 2.4 \times 10^{-15}$ $\mathrm{A}$ is arbitrarily chosen. These plasma parameters are chosen because they are relevant to the Magnetized Dusty Plasma Experiment (Thomas et al. 2012). 
Applying (2.2) and (2.3) to the definitions specified by (2.1) results in a single differential equation for $q(t)$ :

$$
\frac{\mathrm{d} q(t)}{\mathrm{d} t}=-e 4 \pi a^{2} n_{e} \frac{v_{t h, e}}{2 \sqrt{\pi}} \exp \left(\frac{e q(t)}{C k_{b} T_{e}}\right)+e 4 \pi a^{2} n_{i} \frac{v_{t h, i}}{2 \sqrt{\pi}}\left(1-\frac{e q(t)}{C k_{b} T_{i}}\right)+I_{\mathrm{ph}} \mathrm{H}(x(t))
$$

where $\mathrm{H}(x(t))$ is the Heaviside step function, and $x(t)$ is the time-dependent grain position along the inhomogeneity direction, $\hat{x}$.

\section{Numerical approach to charge collection to spherical granule}

We compute the single-grain trajectory in a dust-absent plasma using a stationary inertial lab frame and examine the case of the abrupt inhomogeneity described in Sec. 2. The equations of motion for the dust grain are from the Lorentz force equation:

$$
\begin{gathered}
m_{d} \frac{\mathrm{d} v_{x}}{\mathrm{~d} t}=q(t) B v_{y}, \\
m_{d} \frac{\mathrm{d} v_{y}}{\mathrm{~d} t}=-q(t) B v_{x} .
\end{gathered}
$$

A symplectic, leapfrog integrator is used to solve the equations of motion in (3.1) and (3.2). There are approximately 2000 Newton timesteps (2000 $\left.\Delta t_{N}\right)$ per gyro-cycle, with the Newton timestep held constant during the simulation. The analytical currents for the OML charging model (2.1) are applied to the dust grain using $\Delta q_{n}=I_{\text {tot }} \Delta t_{c}$, where $\Delta t_{c}$ is the charging timestep. This is an adaptive timestep to ensure that the nonlinear differential equation for the grain charge is solved correctly in accordance with the OML model (2.5), simulataneously with the coupled differential equations for the grain motion from the Lorentz force. At the beginning of the simulation, $\Delta t_{c}$ is calculated according to $\Delta t_{c}=e /\left|I_{\text {tot }}\right|$, which is the time for the grain to gain or lose one electron. If $\Delta t_{c}>\Delta t_{N}$, then $q(t)$ is held fixed at its current value and a timer is started with $t_{\mathrm{acc}}=\Delta t_{N}$. This timer, $t_{\mathrm{acc}}$, keeps track of the accumulated time that has passed since the last charge update. During subsequent timesteps, if $t_{\text {acc }}<\Delta t_{N}$, then another Newton timestep is added to $t_{\mathrm{acc}}$. In other words, if $t_{\text {acc }}<\Delta t_{c}$, then $t_{\mathrm{acc}}=t_{\mathrm{acc}}+\Delta t_{N}$. When the condition $t_{\mathrm{acc}} \geqslant \Delta t_{N}$ is satisfied, then one electron is added or subtracted to $q(t)$, depending on the sign of $I_{\text {tot }}$, and the accumulated time is set to $t_{\text {acc }}=\Delta t_{N}-\operatorname{Remainder}\left(\Delta t_{c} / \Delta t_{N}\right)$. This way, when $\Delta t_{c}$ is calculated from the new value of $I_{\text {tot }}$ at the next Newton timestep, the exact value since the last charge update is known.

For this numerical scheme, $q(t)$ is only recorded at the end of every Newton timestep. The total current becomes very small when the grain surface is near the in-situ equilibrium grain charge, and $\Delta t_{c}$ becomes large. The grain fluctuates between neighboring charge states because, in general, $C V_{d}$ is not an exact integer multiple of the elementary charge, $e$. The discrete nature of the particle charging and the adaptive timestep are evident in the step-like plot of Fig. 3.

The charging rate can also be arbitrarily controlled in this model by introducing an adjustable charging parameter, $\alpha$, used in the following way: $q_{n+1}=q_{n}+\Delta q_{n}^{\prime}$, where $\Delta q_{n}^{\prime}=\alpha \Delta q$ as is done in Koepke et al. (2013). This is equivalent to calculating $\Delta t_{c}=\frac{e}{\alpha I_{\text {tot }}}$. For $\alpha=1$, the dust grain charges without restriction, while $\alpha<1$ implies that the grain charges more slowly. This adjustable charging rate parameter is discussed here only insofar as $\alpha$ can be used as a possible way of changing the value 


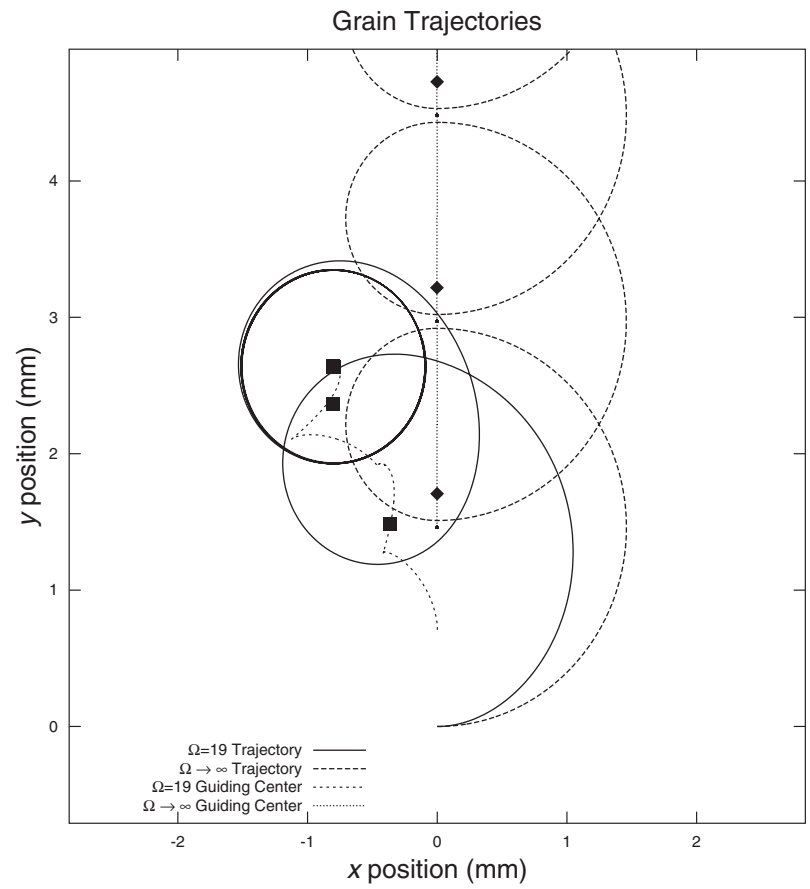

FIGURE 1. Grain trajectories for $a=0.05 \mu \mathrm{m}$. The dashed and solid lines represent the trajectories of $\Omega=\tau_{g} / \tau_{c h}$ ratios for $\Omega \rightarrow \infty\left(10^{16} \mathrm{~m}^{-3}\right)$ and $\Omega=19.204$ (for $n=10^{13} \mathrm{~m}^{-3}$ ) respectively. Squares and diamonds indicate the gyro-averaged guiding centers $\Omega=\tau_{g} / \tau_{c h}$ ratios for $\Omega \rightarrow \infty$ and $\Omega=19.204$ respectively.

of $\tau_{c h}$ in simulations when other plasma parameters, such as the background plasma density, $n_{0}$, are fixed.

\section{Simulation results}

Figure 1 shows a comparison of different grain trajectories for the abrupt, UV inhomogeneity using the OML model with instantaneous and non-instantaneous grain charging. Being negatively charged, the grain gyrates around $\boldsymbol{B}=B \hat{z}$ in a counterclockwise direction. Note how different the instantaneous-charging trajectory is compared with the non-instantaneous-charging trajectory.

The dashed trajectory in Fig. 1 is the trajectory of a dust grain that experiences a step function inhomogeneity in UV illumination (UV light exists for $x>0$, while it is absent for $x<0)$ and instantaneous charging $\left(\tau_{g} \gg \tau_{c}\right)$, or $\Omega=\tau_{g} / \tau_{c h} \rightarrow \infty$. For the portion of the grain's gyro-orbit illuminated by UV $(x>0)$, there is a photoelectric current of electrons in addition to the plasma electron and ion currents that cause the grain charge to become less negative (instantaneously reaching this new charge state $q_{2}$ ). A smaller negative grain charge corresponds to a larger gyro-radius for the grain. Each returns to the shadowed region, $(x<0)$, cuts off the photoelectric current, and the grain instantaneously recovers to a more negative charge state. This repeating pattern of abrupt modulation of charge state results in concatenated semicircles, which, in turn, give rise to a particle drift in the $\hat{y}$-direction that is mutually perpendicular to the equilibrium-charge-inhomogeneity direction and the background magnetic field. This drift is referred to as the grad-q drift $\left(\boldsymbol{v}_{\nabla q}\right)$. Approximating the change to the gyro-radius $R_{L d}=\frac{m_{d} v_{\perp}}{q B}$ to occur abruptly at $\theta=-\pi / 2,3 \pi / 2, \ldots$, which is a good assumption for instantaneous grain charging and $x_{g c}=x(t=0)$, the grad-q 
drift for this particle can be approximated by

$$
\boldsymbol{v}_{\nabla q}=2 \frac{R_{L, 1}-R_{L, 2}}{\tau_{1} / 2+\tau_{2} / 2} \hat{y}=2 \frac{v_{\perp}}{\pi} \frac{\left|q_{1}\right|-\left|q_{2}\right|}{\left|q_{1}\right|+\left|q_{2}\right|} \hat{y}
$$

where $\tau=2 \pi m_{d} /(q B)$ is the gyro-period and the subscripts 1 and 2 indicate equilibrium values in the UV-present and UV-absent regions respectively. Knowing that $v_{\perp}=0.124 \mathrm{~ms}^{-1}$, the equilibrium charge in the shadowed region is $q_{1}=$ $-143.5 e \pm 0.5 e$ and the equilibrium charge in the UV region is $q_{2}=-69.5 e \pm 0.5 e$, the variable charge drift is predicted to be $0.02746 \mathrm{~ms}^{-1}$, which closely matches the simulation result of $0.02743 \mathrm{~ms}^{-1}$.

In Fig. 1, the solid trajectory is the resulting trajectory of a dust grain that has a step function inhomogeneity in UV illumination and non-instantaneous charging. With non-instantaneous charging, the dust grain does not immediately reach the new in-situ equilibrium charge state, and the particle trajectory is modified. In addition to the grad-q drift, the dust grain drifts along the inhomogeneity direction ( $-x$-direction); we refer to this drift component as the gyro-phase drift. Since the gyro-center of the dust grain is drifting along the inhomogeneity direction, the dust grain samples the UV illumination region less with each gyro-orbit, causing the grain to drift out of the boundary associated with the inhomogeneity, and the guiding center drift velocity in the $-x$-direction decreases until it becomes zero and the gyro-center becomes stationary. This drift component along the inhomogeneity direction is a direct result of the finite charging rate of the dust grain in the model.

\section{Theoretical predictions for grad-q and gyro-phase drifts}

For the case of abrupt UV inhomogeneity and instantaneous charging, $(\Omega \rightarrow \infty)$, the grad-q drift is given by (4.1). Since the grain instantaneously reaches the insitu equilibrium grain charge, the grain velocity as a function of time for the first gyro-cycle can be written as

$$
\boldsymbol{v}=\left\{\begin{array}{lll}
v_{x}=v_{\perp} \cos \left(\omega_{2} t\right) \hat{x} & v_{y}=v_{\perp} \sin \left(\omega_{2} t\right) \hat{y} & 0<t<\frac{\pi}{\omega_{2}} \\
v_{x}=v_{\perp} \cos \left(\omega_{1} t-\frac{\omega_{1}+\omega_{2}}{\omega_{2}} \pi\right) \hat{x} & v_{y}=v_{\perp} \sin \left(\omega_{1} t-\frac{\omega_{1}+\omega_{2}}{\omega_{2}} \pi\right) \hat{y} & \frac{\pi}{\omega_{2}}<t<\frac{\pi}{\omega_{1}}+\frac{\pi}{\omega_{2}}
\end{array}\right.
$$

assuming the grain starts with the same conditions as specified in Sec. 4.

For the case of the abrupt UV inhomogeneity and non-instantaneous charging $(\Omega \neq \infty)$, the particle's velocity as a function of time in the presence of the magnetic field can also be computed analytically. These velocity components, being functions of time, can be integrated to average over a gyro-period and compute the guiding center drift velocity components. If the grain has an equilibrium charge of $q_{1}$ in the region where UV is absent, and the grain has an equilibrium charge of $q_{2}$ where UV is present, the charge on the dust grain as a function of time for the charging-discharging grain can be approximated by familiar equations for a discharging/charging capacitor. To generalize to any abrupt inhomogeneity where a grain transitions from a negative charge state to a less negative charge state, $q_{1}$ can be associated with the charge of the more negative charge state and $q_{2}$ can be associated with the charge of the less negative charge state. For the charging part of the gyro-cycle, when the grain transitions to the UV-absent region, the initial charge is given by $q_{c}(t=0)=q_{2}$, $\theta(t=0)=\pi / 2$ with $v_{x}(t=0)=-v_{\perp}$ :

$$
\left|q_{d}(t)\right|=\left(q_{2}-q_{1}\right) \exp \left(-t / \tau_{d}\right)-q_{2},
$$



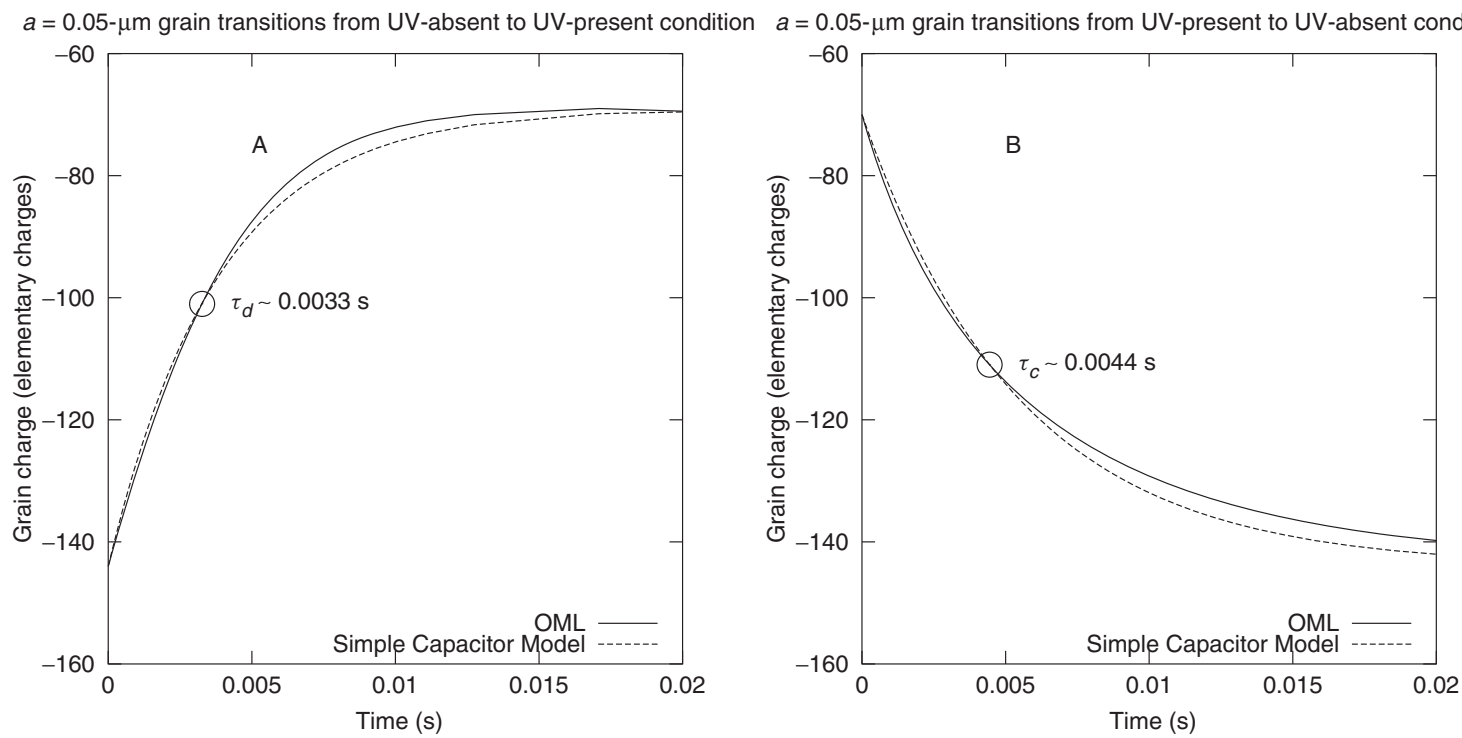

FIGURE 2. Charge evolution for $a=0.05-\mu \mathrm{m}$ grain in the OML and capacitor models. (a) Charge evolution of a dust grain as it transitions from the UV-absent to UV-present region. The UV flux is $4 \times 10^{18} \mathrm{~m}^{-2} \mathrm{~s}^{-1}$, which yields a photoelectron current of $5.03 \times 10^{-15} \mathrm{~A}$. The capacitor model reaches $\frac{1}{e}\left(q_{2}-q_{1}\right)$ at the same time as the OML charge model, although it is a different function of time. (b) Charge evolution of a dust grain as it transitions from the $\mathrm{UV}$-present to UV-absent region.

$$
\left|q_{c}(t)\right|=\left(q_{1}-q_{2}\right) \exp \left(-t / \tau_{c}\right)-q_{1} .
$$

In the above equations, $q_{d}(t)$ and $q_{c}(t)$ are the particle charge as a function of time for the discharging and charging parts of the gyro-cycle. These solutions for the grain charge as a function of time are approximate, since the differential equation for $q(t)$ (2.5) can not be solved analytically. The actual charge evolution in the OML model does not precisely follow (5.2) and (5.3), but it is a reasonable approximation as shown in Fig. 2, which shows the grain charge as a function of time for the discharging and charging portions of the gyro-cycle. Also, these expressions assume that the grain charge is fully charged/discharged during a half gyro-cycle; this restriction can be lifted if $q_{1}=q_{d f}$ in (5.3), where $q_{d f}$ is the charge on the grain when the grain reaches $x=0$, or when $t=t_{d f}$. Using the following definitions: $\omega(t)=\frac{\mathrm{d} \theta}{\mathrm{d} t}=\frac{|q(t)| B}{m_{d}}$, the gyro-phase angle as a function of time can be calculated by integrating $q_{d}(t)$ and $q_{c}(t)$ :

$$
\begin{gathered}
\theta_{d}(t)=\int_{t^{\prime}=0}^{t^{\prime}=t} \frac{\mathrm{d} \theta_{d}\left(t^{\prime}\right)}{\mathrm{d} t^{\prime}} \mathrm{dt} t^{\prime}+\theta_{d}(t=0)=\int_{t^{\prime}=0}^{t^{\prime}=t}\left(\frac{\left|q_{d}\left(t^{\prime}\right)\right| B}{m_{d}}\right) \mathrm{d} t^{\prime}-\frac{\pi}{2}, \\
\theta_{d}(t)=\omega_{2}\left[\tau_{d}\left(\frac{q_{2}-q_{1}}{q_{2}}\right)\left(\exp \left(-\frac{t}{\tau_{d}}\right)-1\right)+t\right]-\frac{\pi}{2}, \\
\theta_{c}(t)=\int_{t^{\prime}=0}^{t^{\prime}=t} \frac{\mathrm{d} \theta_{c}\left(t^{\prime}\right)}{\mathrm{d} t^{\prime}} \mathrm{dt}^{\prime}+\theta_{c}(t=0)=\int_{t^{\prime}=0}^{t^{\prime}=t}\left(\frac{\left|q_{c}\left(t^{\prime}\right)\right| B}{m_{d}}\right) \mathrm{d} t^{\prime}+\theta_{d f}, \\
\theta_{c}(t)=\omega_{2}\left[\tau_{c}\left(\frac{q_{1}-q_{2}}{q_{1}}\right)\left(\exp \left(-\frac{t}{\tau_{c}}\right)-1\right)+t\right]+\theta_{d f},
\end{gathered}
$$

where $\theta_{c}(t=0)=\theta_{d f}$ is the gyrophase angle of the grain when $x=0$, after completing the first half of the gyro-orbit. The general $\theta(t)$ can be defined during the first 
gyro-cycle as follows:

$$
\theta(t)=\left\{\begin{array}{l}
\theta_{d}(t) x>0 \\
\theta_{c}(t) x<0
\end{array} .\right.
$$

With this definition of $\theta$, and $v(t=0)=v_{\perp} \hat{x}$, the general solution for the velocity of the dust grain that satisfies differential equations (3.1) and (3.2) is given by

$$
\begin{aligned}
& v_{x}(t)=v_{0 x} \cos (\theta(t)-\theta(t=0))-v_{0 y} \sin (\theta(t)-\theta(t=0)), \\
& v_{y}(t)=v_{0 y} \cos (\theta(t)-\theta(t=0))+v_{0 x} \sin (\theta(t)-\theta(t=0)),
\end{aligned}
$$

where $v_{0 x}$ and $v_{0 y}$ are the initial velocities of the dust grain in $x$ and $y$ directions respectively. These equations can be applied to the specific conditions described in Sec. 2 for the charging and discharging portions of the gyro-cycle:

$$
\begin{aligned}
& v_{x}(t)=\left\{\begin{array}{ll}
v_{x d}(t)=v_{\perp} \cos \left(\theta_{d}(t)+\frac{\pi}{2}\right) & 0 \leqslant t \leqslant t_{d f} \\
v_{x c}(t)=\begin{array}{ll}
v_{x d}\left(t=t_{d f}\right) \cos \left(\theta_{c}(t)-\theta_{d f}\right) \\
-v_{y d}\left(t=t_{d f}\right) \sin \left(\theta_{c}(t)-\theta_{d f}\right)
\end{array} & t_{d f} \leqslant t \leqslant t_{d f}+t_{c f}
\end{array},\right. \\
& v_{y}(t)=\left\{\begin{array}{ll}
v_{y d}(t)=v_{\perp} \cos \left(\theta_{d}(t)+\frac{\pi}{2}\right) & 0 \leqslant t \leqslant t_{d f} \\
v_{y c}(t)=\begin{array}{l}
v_{y d}\left(t=t_{d f}\right) \cos \left(\theta_{c}(t)-\theta_{d f}\right) \\
+v_{x d}\left(t=t_{d f}\right) \sin \left(\theta_{c}(t)-\theta_{d f}\right)
\end{array} & t_{d f} \leqslant t \leqslant t_{d f}+t_{c f}
\end{array} .\right.
\end{aligned}
$$

To find the trajectory of the dust grain, the components of $v$ must be integrated with respect to time. When putting (5.5) and (5.7) into (5.11) and (5.12), it is clear that the integrals of the form $\cos \left(b\left(\mathrm{e}^{-x}-1\right)+x+\phi\right)$ cannot be integrated analytically, so a numerical approach is necessary. To obtain $x(t)$ and $y(t)$, trapezoidal integration is used. Likewise, to find the guiding center drift velocity components, it is sufficient to numerically integrate:

$$
\begin{aligned}
& v_{x g c}=\frac{1}{\tau_{g}} \int_{t^{\prime}=0}^{t^{\prime}=t_{d f}} \mathrm{dt}^{\prime} v_{x}\left(t^{\prime}\right)+\frac{1}{\tau_{g}} \int_{t^{\prime}=0}^{t^{\prime}=t_{c f}} \mathrm{dt}^{\prime} v_{x}\left(t^{\prime}\right), \\
& v_{y g c}=\frac{1}{\tau_{g}} \int_{t^{\prime}=0}^{t^{\prime}=t_{d f}} \mathrm{dt}^{\prime} v_{y}\left(t^{\prime}\right)+\frac{1}{\tau_{g}} \int_{t^{\prime}=0}^{t^{\prime}=t_{c f}} \mathrm{dt}^{\prime} v_{y}\left(t^{\prime}\right),
\end{aligned}
$$

where $v_{x g c}$ and $v_{y g c}$ are the $x$ and $y$ guiding center velocity as a function of time, $t_{d f}$ is the time during the discharging part of the gyro-cycle at which the grain reaches the transition region $(x=0), t_{c f}$ is the time at which the grain reaches $\theta_{c}=3 /(2 \pi)$, and $\tau_{g}=t_{d f}+t_{c f}$ is the time to complete the entire gyro-orbit (the gyro-period). The gyro-period is defined simply as the time taken to complete one rotation in gyro-phase, and is not necessarily a trivial function of $\pi / \omega_{1}+\pi / \omega_{2}$ as it was for the instantaneous case (5.1). Calculation of these integrals yields a guiding center drift of $v_{x g c}=-0.009 \mathrm{~ms}^{-1}$ and $v_{y g c}=0.0229 \mathrm{~ms}^{-1}$, which is close to the simulation result of $v_{x g c}=-0.0088 \mathrm{~ms}^{-1}$ and $v_{y g c}=0.0227 \mathrm{~ms}^{-1}$. It is also clear from (5.9) and (5.10) that $v_{x}$ and $v_{y}$ are strictly proportional to $v_{0 x}$ or $v_{0 y}$, demonstrating that the guiding center velocities can be normalized by $v_{\perp}=\sqrt{v_{0 x}^{2}+v_{0 y}^{2}}$ without losing any physical insight.

The simulated and analytically obtained gyro-radii $\left(R_{L d}=\frac{m_{d} v_{\perp}}{q(t) B}\right)$ as a function of gyro-phase angle $\theta$ are plotted in Fig. 3. As evident in these figures, the simulation result compares very closely with the analytical prediction based on a capacitor approximation of the OML charge model. 


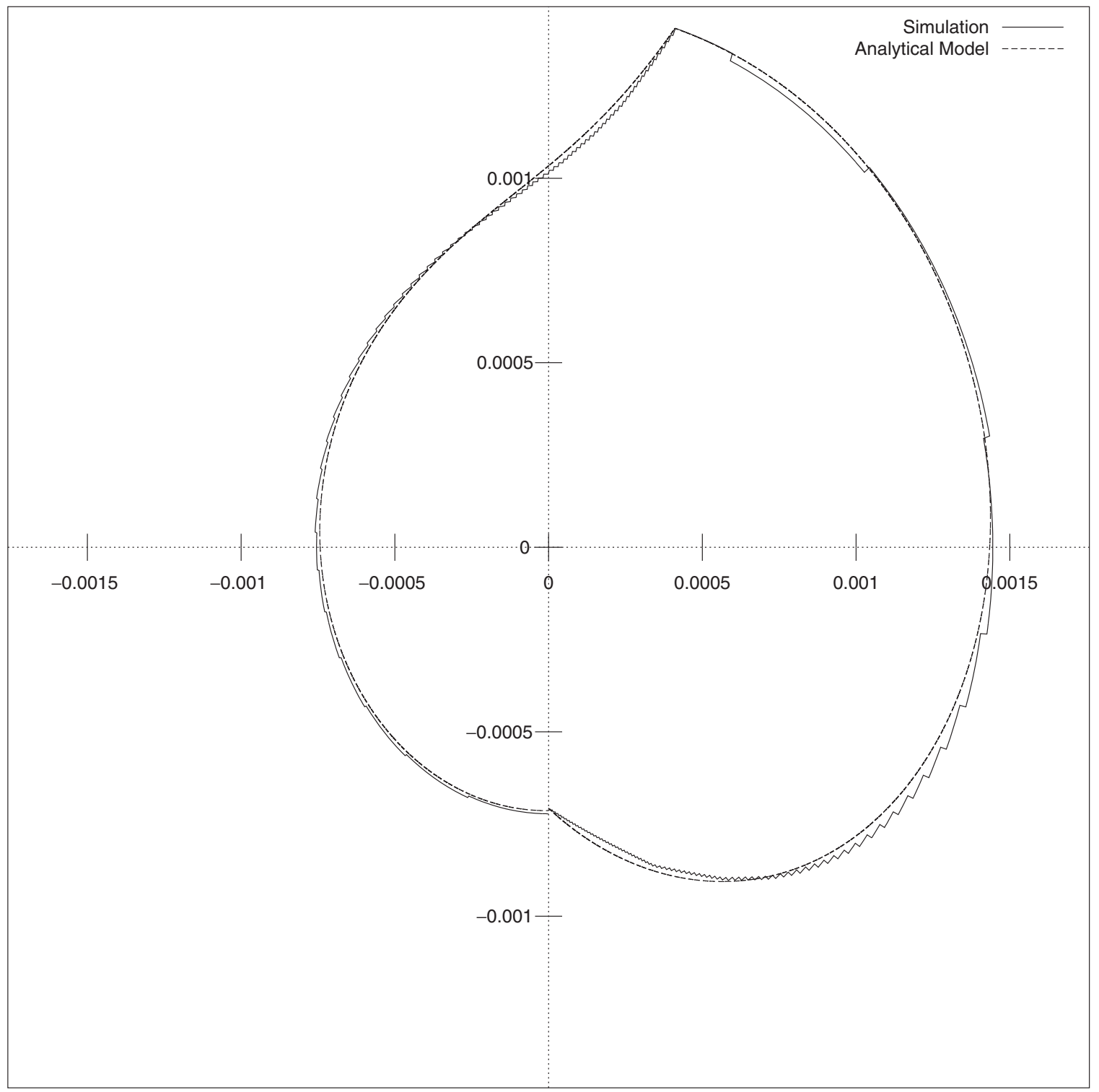

FiguRE 3. Radial distance from the origin is the gyro-radius as a function of gyro-angle. The grain size is $a=0.05 \mu \mathrm{m}$. In the analytical model, $q(t)$ is continuous, while $\frac{\mathrm{d} q}{\mathrm{~d} t}$ is discontinuous at $0^{\circ}$ and $\sim 80^{\circ}$ because the current to the grain abruptly changes at both of these phase angles.

\section{Conclusions}

Gyro-motion within an abruptly inhomogeneous plasma induces charge-state modulation which leads to grad-q and gyro-phase drifts. Because the gyro-phase drift causes the grain's gyro-center to drift along the inhomogeneity direction, dust grains that experience an abrupt plasma inhomogeneity with non-instantaneous charging eventually leave a region of structured inhomogeneity and both grad-q and gyro-phase drift cease. An analytical model is employed here to arrive at general and specific predictions of a dust grain trajectory that experiences an abrupt inhomogeneity in UV illumination.

Because the gyro-phase drift causes micro-particles or nanoparticles to change trajectory, this effect might be used as a means of steering small grains $(<100-\mathrm{nm}$ diameter) in plasma environments where there are magnetized orbit dust grains 
(plasma processing, tokamaks, etc.). If the spatially abrupt UV inhomogeneity is changed to an abrupt amplitude modulated UV inhomogeneity in time, and modulated at a frequency close to gyro-period of specific size grains, these grains can be moved in the $x-y$ plane. Consider for example a 30-nm grain that has $\sim 50$ electrons, and a gyro-period of $\omega=48.7 \mathrm{rads}^{-1}$ for $B=0.0875 \mathrm{~T}$, which is a standard field used in electron cyclotron resonance (ECR) plasmas, where the electron cyclotron frequency is given by $f_{c e}=2.45 \mathrm{GHz}$. For a $30-\mathrm{nm}$ grain population having $T_{d}=T[A r]^{+}=1 /$ $40 \mathrm{eV}$, the thermal speed of dust grains is $0.75 \mathrm{~ms}^{-1}$, corresponding to a gyro-radius of $1.5 \mathrm{~cm}$. If there is a UV source of sufficient intensity to cause the grains to reach the less negative charge state of 25 electrons, the grains will undergo a pure grad-q drift $\left(\tau_{c h} \ll \tau_{g}\right)$ if a modulation frequency of $5.8 \mathrm{~Hz}$ is chosen. Assuming that the phases of the $30-\mathrm{nm}$ grains are randomized, the grains will ultimately leave the plasma volume with a drift direction that is perpendicular to the background magnetic field.

\section{Acknowledgements}

Funding through the US Department of Energy, Office of Fusion Energy Science grant DE-SC0001939 and useful discussions with Dr. Marlene Rosenberg are gratefully acknowledged.

\section{REFERENCES}

Allen, J. E. 1992 Probe theory - the orbital motion approach. Phys. Scr. 45(5), 497.

Colwell, Joshua E., Gulbis, Amanda A. S., Hornyi, M. and Robertson, S. 2005 Dust transport in photoelectron layers and the formation of dust ponds on eros. Icarus 175(1), 159-169.

Dove, A., Horanyi, M., Wang, X., Piquette, M., Poppe, A. R. and Robertson, S. 2012 Experimental study of a photoelectron sheath. Phys. Plasmas 19(4), 043502.

Goree, J. 1994 Charging of particles in a plasma. Plasma Sources Sci. Technol. 3(3), 400.

Koepke, M. E., Walker, J. J., Zimmerman, M. I., Farrell, W. M. and Demidov, V. I. 2013 Signature of gyro-phase drift. J. Plasma Phys.

Northrop, T. G. and Hill, J. R. 1983 The adiabatic motion of charged dust grains in rotating magnetospheres. J. Geophys. Res. Space Phys. 88(A1), 1-11.

Rosenberg, M. and Mendis, D. A. 1995 Uv-induced Coulomb crystallization in a dusty gas. IEEE Trans. Plasma Sci. 23(2), 177-179.

Sickafoose, A. A., Colwell, J. E., Horányi, M. and Robertson, S. 2000 Photoelectric charging of dust particles in vacuum. Phys. Rev. Lett. 84, 6034-6037.

Thomas, E., Merlino, R. L. and Rosenberg, M. 2012 Magnetized dusty plasmas: the next frontier for complex plasma research. Plasma Phys. Control. Fusion 54(12), 124034. 